\title{
Mechanical Alloying Effect on Structural, Thermal and Mechanical Properties of Sn-Bi Alloy
}

\author{
T.T. Issa ${ }^{a, *}$, S.M. KhaleL ${ }^{b}$ And Y.A. ABD AlrazZAQ \\ ${ }^{a}$ Department of Physics, College of Science, University of Baghdad, Baghdad, Iraq \\ ${ }^{b}$ Ministry of Industry and Mineral, Corporation Research and Industrial Development, \\ Chemical and Petrochemical Research Center, Baghdad, Iraq
}

\begin{abstract}
Tin and bismuth powders were used to prepare the Sn40-Bi60 [wt\%] mixture. The mixed powders were mechanically alloyed by processing in the high speed milling machine for 2,4 , and $6 \mathrm{~h}$, respectively. When the milling process had been completed, all the resulting material was annealed and analyzed by XRD. The comparision of the results show, that the alloy is fully formed after 6 hours milling time. Differential scanning calorimeter was used to show the uniformity of the prepared alloy. SEM micrographs reflect the micro structure of the specimens. EDS analysis unveils that the alloy phase is very close to the assumed Sn40-Bi60 [wt\%]. Mechanical properties evaluated by the micro hardness show the best results for $6 \mathrm{~h}$ milling time and $8 \mathrm{~h}$ annealing at $100^{\circ} \mathrm{C}$.
\end{abstract}

DOI: 10.12693/APhysPolA.135.1110

PACS/topics: mechanical alloying, structure, thermal, mechanical, Sn-Bi alloy

\section{Introduction}

The most common alloys used as a low melting point solder are $\mathrm{Sn}-37 \mathrm{~Pb}$ and $\mathrm{Sn}-43 \mathrm{~Pb}-14 \mathrm{Bi}$. Tin solder has several attributes that are not desirable for certain applications, according to the regulations of the EU Waste Electrical and Electronic Equipment Directive (WEEE). The restriction of hazardous substances evokes much interest in lead-free solders [1], based on other elements like bismuth, copper, silver, and indium. The binary alloy tin-bismuth, with low melting point, are used in some low temperature applications [2]. The most common method to produce it was the melt-ingot process. Currently the mechanical alloying (MA) is the progressive technology of manufacturing the necessary alloys with low coast and in commercial quantities. The quality of the product is better, then for the previous methods.

The mechanical alloying is a powder technology was established in 1960 [3]. This method depends mainly on cold welding, fracturing, and reinforcing of powder particles by using high energy ball milling machine. This technique is suitable to produce a different kind of equilibrium and non equilibrium alloy phases [4]. In present work the prepared alloy has uniform crystal structure and microstructure with better thermal behavior and mechanical resistance compared with those prepared by the melting process [5].

\section{Materials and equipment}

The tin and bismuth powders of $99.999 \%$ purity and 120-140 $\mu \mathrm{m}$ grain size were used. There samples of the

\footnotetext{
*corresponding author; e-mail: alazbrh@yahoo.com
}

combination Sn40-Bi60 [wt\%] were inserted to the StSt container of $240 \mathrm{ml}$, with three St-St milling balls of $20 \mathrm{~g}$ in weight. Mechanical alloying was led at $600 \mathrm{rpm}$ speed for milling time 2,4 , and $6 \mathrm{~h}$, respectively. The resulted samples after milling were annealed at $100^{\circ} \mathrm{C}$ for 8 hours in still air, and then tested by X-ray diffraction (XRD) to identify the phase composition. Differential scanning calorimeter (DSC) was used to examine the thermal behavior of prepared alloys. At the heating rate of $5{ }^{\circ} \mathrm{C} / \mathrm{min}$ the beginning, peak, and the end of melting process was established. The microstructure of all samples were investigated by using the scanning electron microscope (SEM) TESCAN VEGA3. The additional EDS analysis delivered the information on the composition of the prepared alloy. The microhardness tests of the materials were performed according to the Vickers method on polished pellets, according to the ASTMB 933 standard.

\section{Results and discussion}

In order to master the mechanical alloying technology of preparation of the solder alloy XRD measurenments were performed. The results obtained for 2 and $4 \mathrm{~h}$ milling time showed only partial formation of the alloy phase (see Fig. 1a, b).

The definitive processing result was achieved at $6 \mathrm{~h}$ milling time, as is shown in Fig. 1c. Alloy phase was detected and distinguished according to the file N1997ICPdS (International Center for Diffraction Data). The patterns unveil the alloy phase with sharply increasing intensity, especially for the alloy milled for $6 \mathrm{~h}$, Fig. 1c. The difference in peak phase intensity $(2 \Theta=$ $\left.39^{\circ}, 40^{\circ}\right)$ is due to the effective decreasing in residual stresses caused by the mechanical alloying (MA), and because of the dissolution of bismuth atoms as a solid solute at the grain boundaries of tin as milling time increases. The results of the XRD test are supported 

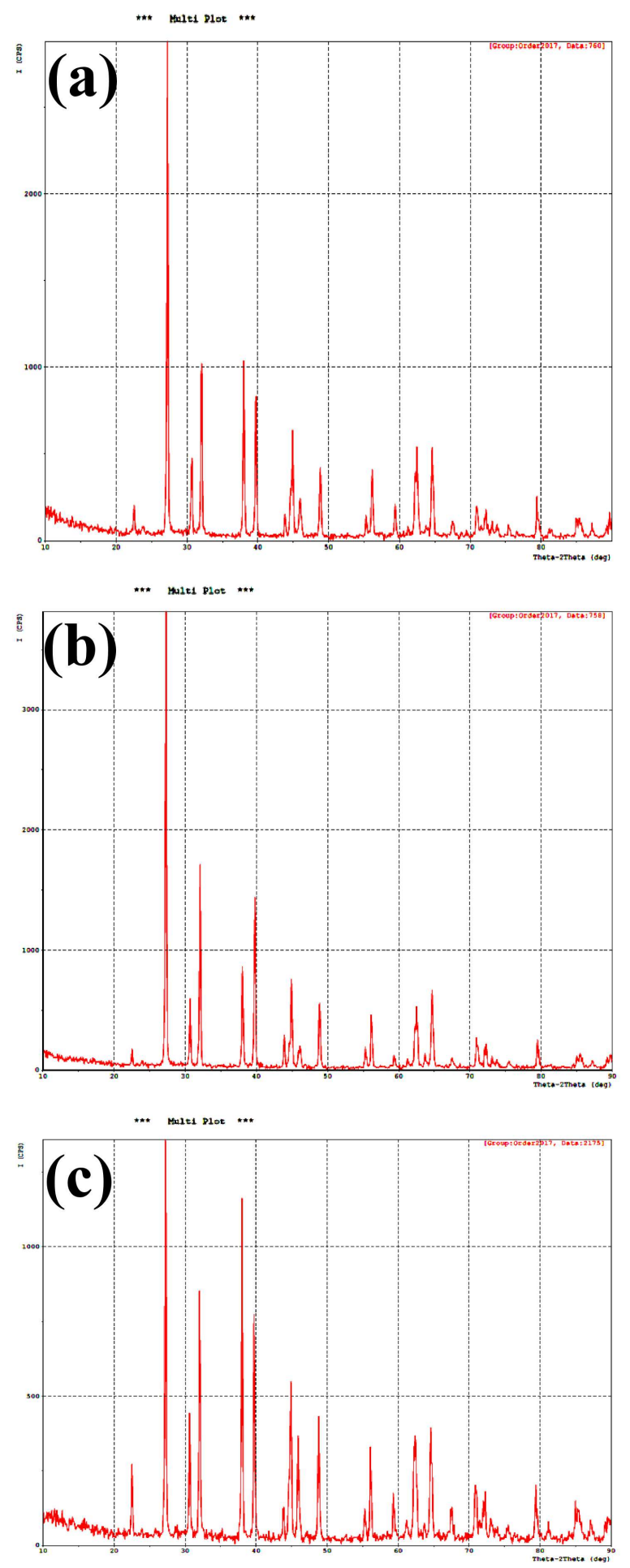

Fig. 1. X-ray diffraction pattern showed the partial formation of alloy phase at (a) $2 \mathrm{~h}$, (b) $4 \mathrm{~h}$, (c) $6 \mathrm{~h}$ milling time (full phase formation).

by the data from DSC. The DSC results are shown in Fig. 2, curves a-c, for the alloys prepared at 2, 4, and $6 \mathrm{~h}$ of milling time, respectively. The discontinuity slope attriburable to the permeating portion (onset) is indicated as $132.55^{\circ} \mathrm{C}, 132.67^{\circ} \mathrm{C}, 132.13^{\circ} \mathrm{C}$, respectively, and the melting start temperature (peak) is indicated at

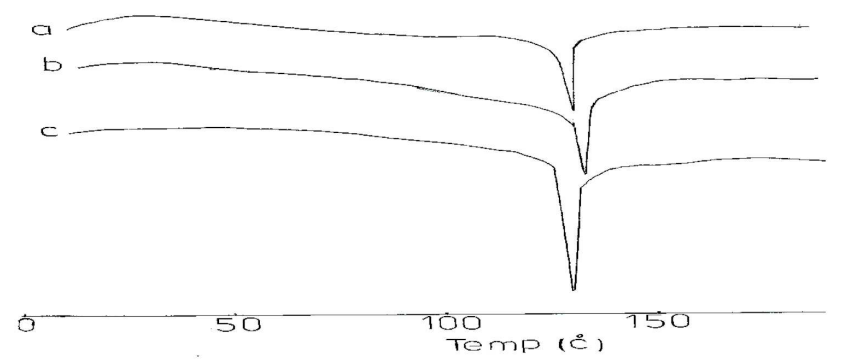

Fig. 2. DSC typical profile for the prepared alloy, (a) $2 \mathrm{~h}$, (b) $4 \mathrm{~h}$, (c) $6 \mathrm{~h}$ milling time.

$135.45^{\circ} \mathrm{C}, 134.53{ }^{\circ} \mathrm{C}, 135.07^{\circ} \mathrm{C}$, respectively. The Sn-Bi melting appears to be associated with the micro structure homogeneity [6].

The microstructure for the prepared alloy is shown in Fig. 3, as SEM micrographs after effective annealing. When the formation of alloy phase is not completed (at 2 and $4 \mathrm{~h}$ milling time), its partialness is evident, as is shown in Fig. 2a and b, respectively. The completed phase with fully homogeneous microstructure is shown in Fig. 2c. The alloy prepared at $6 \mathrm{~h}$ milling time has the
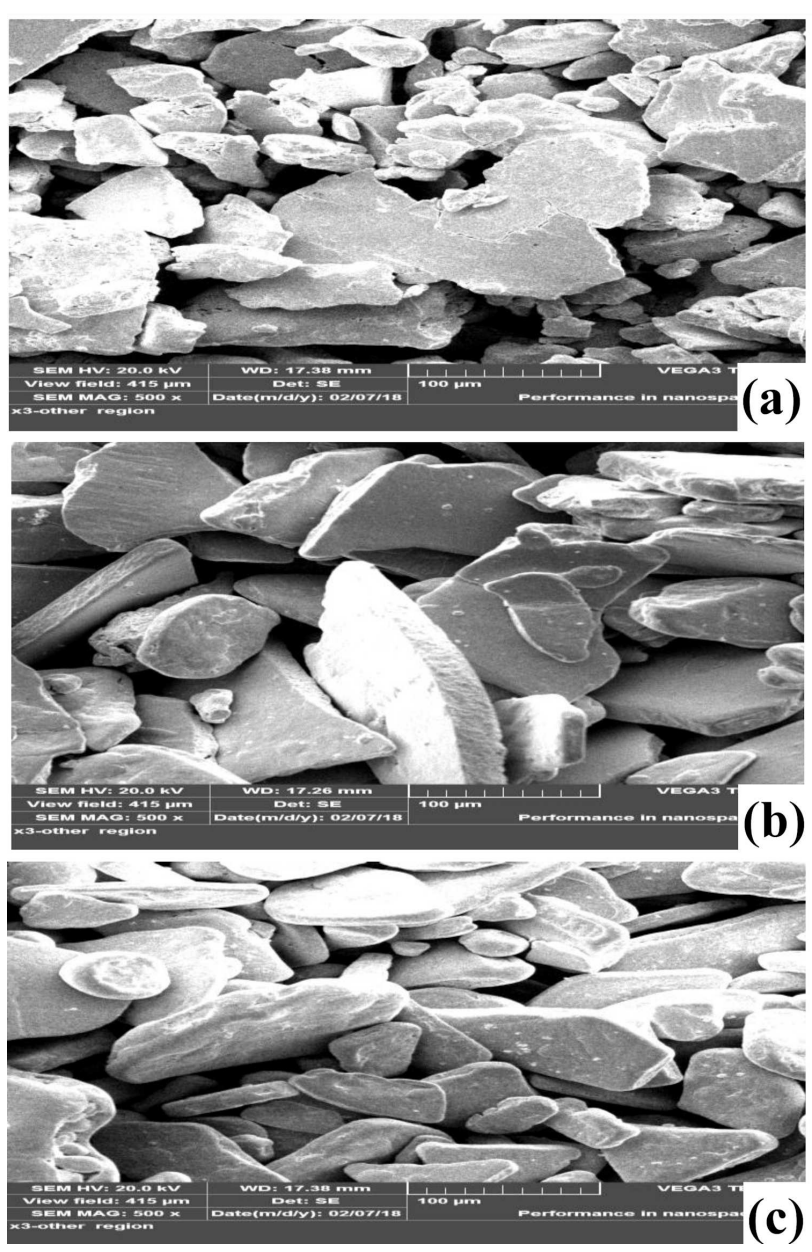

Fig. 3. SEM micrographs of the alloy prepared at: (a) $2 \mathrm{~h}$, (b) $4 \mathrm{~h}$, and (c) $6 \mathrm{~h}$ milling time. 
grain size of $100 \mu \mathrm{m}$, lower than the starting grain size $(120-140 \mu \mathrm{m})$. The remarkable microstructure obtained after annealing is due to the dissolution of the residual stress caused by MA [7].

The EDS analysis for all specimens confirms, that the phase alloy consists of $40 \mathrm{wt} \%$ of tin and 60 [wt\%] of bismuth, as shown in Fig. 4.

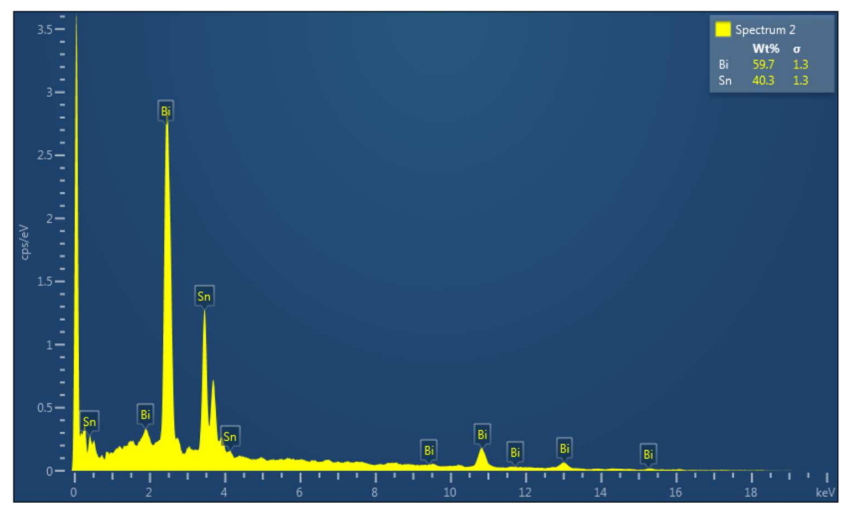

Fig. 4. EDS analysis for the prepared alloy by mechanical alloying.

Results of the Vickers microhardness measurements for all specimens after annealing are shown in Fig. 5. The data confirm, that the alloy prepared at $6 \mathrm{~h}$ milling time has better properties than those prepared at 2 and $4 \mathrm{~h}$ milling time, respectively.

The results confirms the advantages offered by the improvement caused by annealing, which produces the microstructure of higher strength and of greater phase homogeneity.

\section{Conclusion}

Mechanical alloying effect favorably on all the alloys we investigated. The crystal structure, thermal behaviour of the microstructure and mechanical properties for the binary alloy Sn40-Bi60 [wt\%] prepared at different milling time is improved. The effectiveness of the annealing process after the preparing of the alloy is indicated also.

\section{Acknowledgments}

We express our deep gratitude to all, who offered us help and support this work, especially to the employees of the Material Labs in the State Commission for Research and Development, Ministry of Industry and Mineral.

\section{References}

[1] T. Tlssa, F.N. Jasim, H.J. Mohammed, Z.K. Abbas AIP Conf. Proc. 1809, 020024 (2017).

[2] M.L. Huang, C.M.L. Wn, J.K.L. Lai, Y.C. Chan, J. Electr. Mater. 29, 1021 (2000).

[3] M.L. Huang, C.M.L. Wu, J.K.L. Lai, L. Wang, F.G. Wang, J. Mater. Sci. Mater. Electron. ll, 57 (2000).

[4] F. Khodabakhshi, R. Sayyadi, N. Shahamat Javid, Mater. Sci. Eng. C 702, 371 (2017).

[5] S. Amares, M.N.E. Efzan, T.C. Yap, Adv. Mater. Res. 845, 261 (2014).

[6] M. Abtew, G. Selvaury, Mater. Sci. Engin. R 27,95 (2000).

[7] W.-L. Chen, C.-Y. Yu, C.-Y. Ho, J.-G. Duh, Mater. Sci. Eng. A 613, 193 (2014). 